\title{
Silicon photomultiplier detector for atmospheric lidar applications
}

\author{
Jordi Riu, ${ }^{1, *}$ Michaël Sicard ${ }^{2,3}$ Santiago Royo, ${ }^{1}$ and Adolfo Comerón ${ }^{2}$ \\ ${ }^{1}$ Center for Sensors, Instrumentation and Systems Development, Universitat Politècnica de Catalunya, \\ Rambla Sant Nebridi 10, 08222 Terrassa, Spain \\ ${ }^{2}$ Department of Signal Theory and Communications, Remote Sensing Laboratory, Universitat Politècnica de Catalunya, \\ c/ Jordi Girona 1-3, 08034 Barcelona, Spain \\ ${ }^{3}$ Institut d'Estudis Espacials de Catalunya, Universitat Politècnica de Catalunya, c/ Jordi Girona 1-3, 08034 Barcelona, Spain \\ ${ }^{*}$ Corresponding author: jordi.riu@cd6.upc.edu
}

Received January 3, 2012; accepted February 3, 2012;

posted February 8, 2012 (Doc. ID 160770); published March 27, 2012

\begin{abstract}
The viability and performance of using a silicon photomultiplier (SiPM) in atmospheric lidar applications is experimentally compared against the well-established use of photomultiplier tubes. By using a modified lidar setup for simultaneous data acquisition of both types of sensors, we demonstrate that a SiPM can offer appropriate qualities for this specific application where the detection of fast, extremely low light pulses and large dynamic range signals are essential capabilities. The experimental results show that the SiPM has an appropriate behaviour offering suitable capabilities for elastic, backscatter aerosol lidars. To the best of our knowledge, this is the first study showing SiPM for atmospheric lidar applications. (c) 2012 Optical Society of America
\end{abstract}

OCIS codes: $\quad 010.1100,010.3640,040.5250,040.6070,280.3420,280.3640$.

A major problem for all atmospheric lidar systems is that the optical power received from long distances is very low. As a result, highly sensitive sensors are of special interest for this application. In addition, taking into account the nature of the range measurement based in time of flight, a large bandwidth is also important. Another characteristic lies in the large dynamic range of the signals caused by the excess of optical power in short distances and fast loss of backscattered light at longer distances, resulting in a characteristic waveform. Based on these three requirements, the list of useful sensors is very short and mainly restricted to photomultiplier tubes (PMTs) and avalanche photodiodes (APDs).

However, recently developed silicon photomultipliers (SiPMs) could be an alternative to the sensors mentioned above. SiPMs are devices consisting of multiple avalanche photodiode pixels operating in Geiger mode. All of them are joined together in a common substrate under a common load [1] . When photons enter a pixel, a pulse is generated at its output regardless of the number of photons. The output of the detector is the sum of the pulses generated by each pixel, which depends on the amount of optical power detected [2]. The output is also affected by noise which is quantified as a frequency of activated pixels without being fired by a photon.

As far as we know, the use of SiPM for atmospheric lidar application is new. Recently, some studies have been carried out on SiPM as an alternative to PMTs in applications like positron emission tomography (PET) [3], astrophysics particle detection [4], or imaging Cherenkov counters [5]. In this Letter we demonstrate the feasibility of using this kind of sensor for elastic, backscatter aerosol lidars. We also introduce the SiPM as a possible alternative for the well established PMT technology [6].

The lidar from the Universitat Politècnica de Catalunya (UPC) is described in detail in [7]. It is formed by a pulsed Nd:YAG laser with a second and third harmonic generator. The energy per pulse at $532 \mathrm{~nm}$ is about $160 \mathrm{~mJ}$ with duration of $8 \mathrm{~ns}$ and a repetition rate of $20 \mathrm{~Hz}$. The recep- tion part is composed of an $\varnothing 40 \mathrm{~cm}$ telescope with a focal length of $3910 \mathrm{~mm}$. The light is transported from the telescope to the polychromator by an Ø3 $\mathrm{mm}$ fiber bundle. A polychromator was especially designed for the experiment (see Fig. 1). A first group of lenses are used to collimate the beam. A 50\%-50\% beamsplitter is used to separate the backscattered light towards each detector. A couple of focusing lenses were optimally positioned (supported by ray-tracing simulations) in front of the detectors to form an $\varnothing 3 \mathrm{~mm}$ spot on the active area of the detectors. Interference filters centered at $532 \mathrm{~nm}$ were used to block other wavelengths. The polychromator is designed with $\varnothing 25.4 \mathrm{~mm}$ optical components and has a receiving field of view of $\sim 6 \mathrm{mrad}$. The digitalization

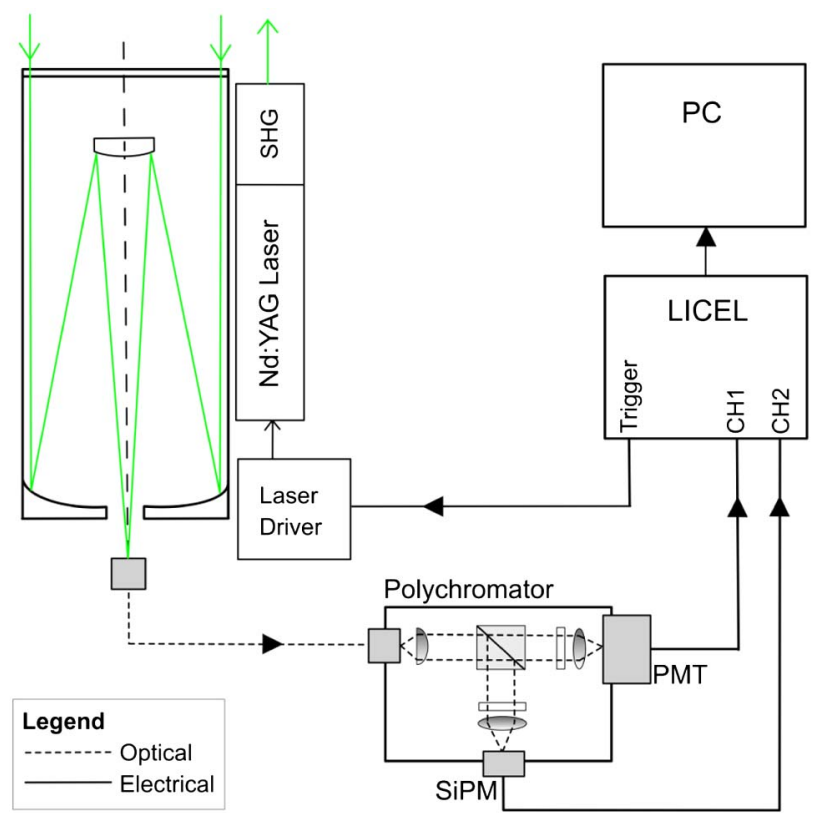

Fig. 1. (Color online) Experimental setup for simultaneous PMT and SiPM measurements. 
of the output of each sensor was made in analog mode by means of a Licel TR20-160 transient recorder. A vertical resolution of $3.75 \mathrm{~m}$ is achieved by a sampling at 40 Msamples/s. The PMT bias voltage was fixed to $-830 \mathrm{~V}$. Licel inputs only accept negative signals, hence special care was taken in the case of the SiPM. Standard reverse bias circuits apply positive voltage at cathode side obtaining positive signals at the output. In our case, the detector was biased applying negative voltage at anode side to ensure reverse bias [8]. An optimum voltage value of $-69.85 \mathrm{~V}$ was found so that the received signal occupies the largest dynamic range of the transient recorder and never saturates. For a good timing resolution [9], sensors work with a load impedance of $50 \Omega$. Both detectors are from Hamamatsu. The SiPM model is S10362-33-100C (Ø3 mm; 900 pixels) and the PMT is R7400P. Table 1 summarizes the parameters of interest of both sensors. The efficiency refers to the photon detection efficiency for the SiPM and the quantum efficiency for the PMT. Note that the dark count parameter is only indicative since it is not used for calculations.

The measurement was performed on October 182011 between 2232 and 2301 universal time coordinate (UTC) with an outside temperature of $18^{\circ}$. The measurement duration was $30 \mathrm{~min}$ and the temporal resolution $1 \mathrm{~min}$ (each profile is an integration of 1200 lidar return pulses). Aerosols were observed up to $3.35 \mathrm{~km}$ above ground level (agl). According to AERONET, the aerosol optical thickness was rather low, around 0.05 at $532 \mathrm{~nm}$. The aerosol backscatter coefficient was retrieved by the twocomponent inversion algorithm [10-12] using a constant lidar ratio of $50 \mathrm{sr}$.

The signal-to-noise ratio (SNR) is shown in Fig. 2 for the $30 \mathrm{~min}$ integrated profile. Below $3.35 \mathrm{~km}$, the SiPM SNR is approximately 1.5 to 1.7 times higher than that of the PMT. In signal shot-noise dominated regime at low altitude, the photodetector SNR can be written as

$$
\mathrm{SNR}^{s n}=\left(\frac{P_{s} \lambda}{2 h c B} \frac{\eta}{F}\right)^{\frac{1}{2}}
$$

where $P_{s}$ is the signal optical power, $\lambda$ the wavelength, $\eta$ the quantum efficiency, $h$ the Planck's constant, $c$ the light speed, $B$ the electrical bandwidth, and $F$ the excess noise factor. For a given signal optical power, the ratio of the SNR of both detectors can be approximated by

$$
\frac{\mathrm{SNR}_{S i P M}^{s n}}{\mathrm{SNR}_{P M T}^{s n}}=\left(\frac{\eta_{S i P M}}{F_{S i P M}} \frac{F_{P M T}}{\eta_{P M T}}\right)^{\frac{1}{2}} \approx 2.32 .
$$

Table 1. Sensor Parameters at the Bias Voltage, Temperature and Wavelength Used for the Experiment

\begin{tabular}{lcc}
\hline & SiPM & PMT \\
\hline Bias voltage & $-69.85 \mathrm{~V}$ & $-830 \mathrm{~V}$ \\
Efficiency & $60 \%$ & $10 \%$ \\
Excess noise factor & 1.42 & 1.30 \\
Dark current & $0.7 \mu \mathrm{A}$ & $4 \mathrm{nA}$ \\
Active area & $9 \mathrm{~mm} 2$ & $50.26 \mathrm{~mm} 2$ \\
Gain & $2.4 \times 10^{6}$ & $1.3 \times 10^{6}$ \\
Dark count & $8 \mathrm{Mcps}$ & $80 \mathrm{cps}$ \\
\hline
\end{tabular}

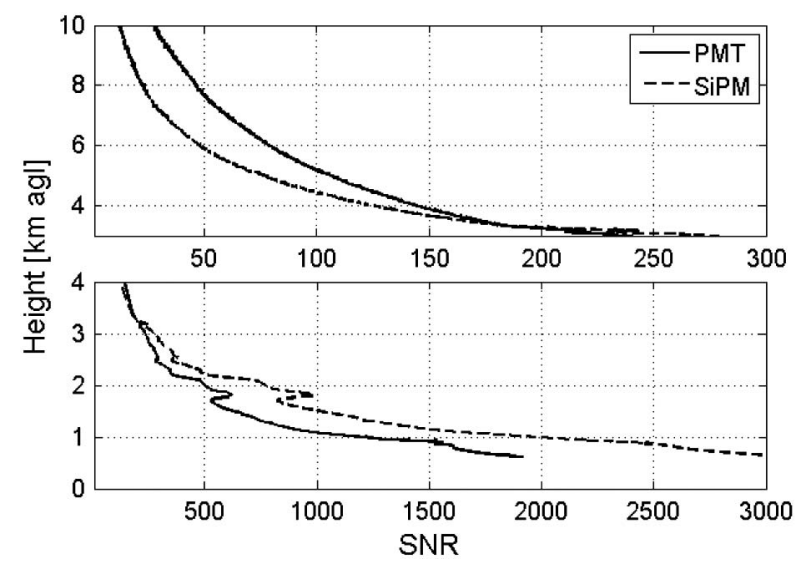

Fig. 2. SNR as a function of height of the PMT and SiPM detectors.

Above $8 \mathrm{~km}$, the PMT SNR is approximately two times higher than that of the SiPM. In photoreceiver-noise dominated regime, which occurs at high altitude, the photodetector SNR can be written as

$$
\mathrm{SNR}^{r n}=\frac{q^{1 / 2} P_{s} \lambda}{h c(2 B)^{1 / 2}}\left(\frac{\eta^{2} M}{F I_{d}}\right)^{\frac{1}{2}},
$$

where $I_{d}$ is the dark current. Because the multiplication factors of both the SiPM and the PMT are very high, in photoreceiver-noise dominated regime, the thermal noise of the $50 \Omega$ load resistor is negligible as compared to the shot dark-current noise. For a given signal optical power, the ratio of the SNR of both detectors can be approximated by

$$
\frac{\mathrm{SNR}_{P M T}^{r n}}{\operatorname{SNR}_{S i P M}^{r m}} \approx \frac{\eta_{P M T}}{\eta_{S i P M}}\left(\frac{F_{S i P M} I_{d, S i P M} M_{P M T}}{F_{P M T} I_{d, P M T} M_{S i P M}}\right)^{\frac{1}{2}} \approx 1.70 .
$$

In both cases, the SNR ratios measured agree well with the theoretical values taking into account the uncertainties of the parameters given by the photodetectors' manufacturer. This analysis shows that, at least in an optically thin atmosphere, the SiPM provides a slightly higher SNR than the PMT in the low region of the atmosphere where aerosols are present. In the free troposphere the SiPM SNR is slightly lower than that of the PMT. A lower SNR in the free troposphere yields an increase of the error of the retrieved backscatter coefficient because of noise affecting the signal at the calibration height, which is inversely proportional to the SNR [13]. In the case presented here, this error would be approximately two times larger for the SiPM than for the PMT.

The aerosol backscatter coefficient profiles retrieved with both detectors are shown in Fig. 3, as well as the time series of the range-square-corrected signal (RSCS). The SiPM signal has been inverted as is the dash line and applying a dead-time correction (dot line). According to the manufacturer the "pulse level recovery" time, i.e., the time period for a pixel to be restored to a state capable of detecting the next photons, is approximately 100-200 ns for our SiPM. We have estimated this time globally (for all pixels) in an empirical way by applying a nonparalyzable dead-time correction to the received power and adjusting 

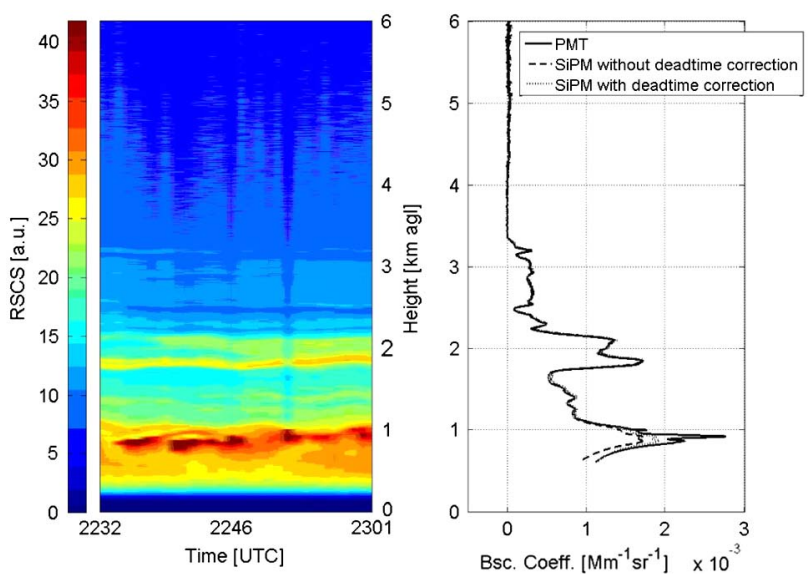

Fig. 3. (Color online) Time series of the RSCS between 2232 and 2301 UTC and backscatter coefficient profiles retrieved with the PMT and SiPM detectors.

the SiPM RSCS to that of the PMT in the first kilometer. The value found is $\sim 0.134 \mathrm{~ns}$, which scaled to one pixel gives $\sim 120$ ns. Figure 3 shows that the profiles overlap perfectly above $1 \mathrm{~km}$. The dead-time effect is clearly visible below $1 \mathrm{~km}$ and its correction significantly improves the agreement between both detectors. However, in view of the difficulties for the SiPM to track the strong and thin layer just below $1 \mathrm{~km}$, we suspect that a response time might make the detector slow to fast changes. Other SiPM models of the same effective area as the one used in this experiment, and more pixels of smaller size and shorter dead time, could ease the need for the dead-time correction.

Apart from the mentioned dead-time correction and a possible slow response time, the SiPM might also suffer from a slower-rising overlap function because of its relatively small size, which makes it more sensitive to misalignments than the PMT. A model of larger size and more pixels would help to cope with the latter problem. Despite these effects, the study shows that the SiPM is suitable for elastic-backscatter aerosol lidars offering a performance comparable to PMTs.
The authors thank the Spanish Ministry of Science and Innovation (MICIIN) for projects DPI2009-13379, and DPI2011-25525, which partially funded this research. The lidar activities are supported by the 7th Framework Programme project Aerosols, Clouds, and Trace Gases Research Infrastructure Network (ACTRIS) (Grant agreement no. 262254) and by the MICINN and FEDER funds under the project TEC2009-09106/TEC, and the Complementary Actions CGL2009-08031-E/CLI, CGL2010-09225-E and CGL2011-13580-E/CLI.

\section{References}

1. K. Yamamoto, K. Yamamura, K. Sato, T. Ota, H. Suzuki, and S. Ohsuka, in Proceedings of the IEEE Nuclear Science Symposium Conference Record (IEEE, 2006), pp. 10941097.

2. P. Eraerds, M. Legré, A. Rochas, H. Zbinden, and N. Gisin, Opt. Express 15, 14539 (2007).

3. A. Nassalski, M. Moszyński, A. Syntfeld-Każuch, T. Szczéśniak, Ł. Świderski, D. Wolski, T. Batsch, and J. Baszak, IEEE Trans. Nucl. Sci. 57, 1008 (2010).

4. N. Otte, B. Dolgoshein, J. Hose, S. Klemin, E. Lorenz, R. Mirzoyan, E. Popova, and M. Teshima, Nucl. Phys. B 150, 144 (2006).

5. P. Buzhan, B. Dolgoshein, L. Filatov, A. Ilyin, V. Kantzerov, V. Kaplin, A. Karakash, F. Kayumov, S. Klemin, E. Popova, and S. Smirnov, Nucl. Instrum. Meth. Phys. Res. A 504, 48 (2003).

6. V. D. Kovaltchouk, G. J. Lolos, Z. Papandreou, and K. Wolbaum, Nucl. Instrum. Meth. Phys. Res. A 538, 408 (2005).

7. D. Kumar, M. Sicard, S. Tomás, C. Muñoz, F. Rocadenbosch, and A. Comerón, Proc. SPIE, 6367 63670U (2006).

8. S. Seifert, H. T. van Dam, J. Huizenga, R. Vinke, P. Dendooven, H. Löhner, and D. R. Schaart, IEEE Trans. Nucl. Sci. 56, 3726 (2009).

9. R. Vinke, H. Löhner, D. R. Schaart, H. T. van Dam, S. Seifert, F. J. Beekman, and P. Dendooven, Nucl. Instrum. Meth. Phys. Res. A 610, 188 (2009).

10. J. D. Klett, Appl. Opt. 24, 1638 (1985).

11. F. G. Fernald, Appl. Opt. 23, 652 (1984).

12. Y. Sasano and H. Nakane, Appl. Opt. 23, 11 (1984).

13. M. Sicard, A. Comerón, F. Rocadenbosch, A. Rodríguez, and C. Muñoz, Appl. Opt. 48, 176 (2009). 